ARTICLE

Received 8 Oct 2013 | Accepted 18 Feb 2014 | Published 24 Mar $2014 \quad$ DOl: 10.1038/ncomms4469

\title{
Mutations in epigenetic regulators including SETD2 are gained during relapse in paediatric acute lymphoblastic leukaemia
}

Brenton G. Mar ${ }^{1,2}$, Lars B. Bullinger ${ }^{3}$, Kathleen M. McLean ${ }^{1}$, Peter V. Grauman ${ }^{4}$, Marian H. Harris ${ }^{5}$, Kristen Stevenson ${ }^{6}$, Donna S. Neuberg ${ }^{6}$, Amit U. Sinha7 , Stephen E. Sallan ${ }^{1,2}$, Lewis B. Silverman ${ }^{1,2}$, Andrew L. Kung ${ }^{8}$, Luca Lo Nigro9 ${ }^{9}$, Benjamin L. Ebert ${ }^{4} \&$ Scott A. Armstrong ${ }^{7}$

Relapsed paediatric acute lymphoblastic leukaemia (ALL) has high rates of treatment failure. Epigenetic regulators have been proposed as modulators of chemoresistance, here, we sequence genes encoding epigenetic regulators in matched diagnosis-remission-relapse ALL samples. We find significant enrichment of mutations in epigenetic regulators at relapse with recurrent somatic mutations in SETD2, CREBBP, MSH6, KDM6A and MLL2, mutations in signalling factors are not enriched. Somatic alterations in SETD2, including frameshift and nonsense mutations, are present at $12 \%$ in a large de novo ALL patient cohort. We conclude that the enrichment of mutations in epigenetic regulators at relapse is consistent with a role in mediating therapy resistance.

\footnotetext{
${ }^{1}$ Department of Pediatric Oncology, Dana Farber Cancer Institute, Boston, Massachusetts 02215, USA. ${ }^{2}$ Department of Hematology/Oncology, Boston Children's Hospital, Boston, Massachusetts 02115, USA. ${ }^{3}$ Department of Internal Medicine III, University of Ulm, Ulm 89081, Germany. ${ }^{4}$ Division of Hematology, Department of Medicine, Brigham and Women's Hospital, Harvard Medical School, Boston, Massachusetts 02115, USA. ${ }^{5}$ Department of Pathology, Boston Children's Hospital, Boston, Massachusetts 02115, USA. ${ }^{6}$ Biostatistics \& Computational Biology, Dana Farber Cancer Institute, Boston, Massachusetts 02215, USA. ${ }^{7}$ Human Oncology and Pathogenesis Program and Department of Pediatrics, Memorial Sloan-Kettering Cancer Center, New York, New York 10065, USA. ${ }^{8}$ Department of Pediatrics, Columbia University Medical Center, New York, New York 10032, USA. ${ }^{9}$ Oncoematologia Pediatrica, Azienda Policlinico OVE, 95125, Catania, Italy. Correspondence and requests for materials should be addressed to S.A.A. (email: armstros@mskcc.org).
} 


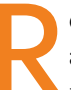
elapse occurs in $15-20 \%$ of paediatric ALL and $50 \%$ of adult ALL patients. Compared to de novo leukaemia, relapsed leukaemia is chemoresistant and long-term survival is poor; however, the genetic basis for this is not well understood. Studies of matched diagnosis and relapse ALL samples by SNP arrays demonstrated that $92 \%$ of relapsed ALL had DNA copy number variant (CNV) changes from diagnosis. Surprisingly, over half of relapses are missing CNVs present in the predominant clone at diagnosis and appear to have derived from a minor subclone in many cases ${ }^{1}$. Targeting alterations that are frequently gained at relapse is therefore a rational therapeutic strategy, as these lesions are likely associated with chemotherapy resistance and clonal survival. Unfortunately, most CNV alterations gained at ALL relapse are difficult to target, such as CDKN2A or ETV6 deletion.

Alterations in epigenetic regulators have recently been identified in genome and exome sequencing studies of chemotherapy resistant, very high-risk subsets of de novo ALL. For example, $48 \%$ of early T-cell precursor (ETP) $\mathrm{ALL}^{2}$ and $60 \%$ of hypodiploid $\mathrm{ALL}^{3}$ have somatic mutations in genes encoding epigenetic regulators. In addition, loss-of-function mutations in the histone acetyltransferase CREBBP have also been described in diagnostic samples from ALL patients who relapse, and in samples from relapsed patients ${ }^{4}$. As the enzymatic activities of CREBBP are directly opposed by histone deacetylases, this provided one possible rationale for why histone deacetylase inhibitors have activity in ALL. These studies suggest a broader paradigm in that mutations in epigenetic regulators may be associated with therapy resistance and can be targeted indirectly through inhibition of the enzymatic activity of opposing regulators. However, thus far, no published study has addressed the mutational frequency of epigenetic regulators at ALL relapse, and whether they are frequently enriched at relapse compared to at diagnosis.

Here, we sequence matched sets of diagnosis-remissionrelapse samples from paediatric ALL patients, and find a significant enrichment of mutations in epigenetic regulators at relapse, including the $\mathrm{H} 3 \mathrm{~K} 36$ trimethyltransferase SETD2. We conclude that these mutations are likely involved in chemotherapy resistance and deserve further study to define their role in the prognosis and treatment of ALL.

\section{Results}

Somatic mutations in epigenetic regulators in de novo ALL. To study this, we performed custom exon-hybrid capture and Illumina sequencing of 472 known and putative epigenetic regulators and frequently mutated genes in leukaemia (Supplementary Table 1) on matched diagnostic-remission samples from 60 paediatric B-ALL patients, and matched relapse samples from 30 of those patients that relapsed. Patients in this cohort were those with paediatric ALL (primarily B cell), with a range of cytogenetics and risk groups (Supplementary Table 2). In this cohort, relapsed patients had the bone marrow as a site of relapse, with over $60 \%$ marrow involvement. $70 \%$ of patients had an identified somatic mutation at diagnosis and/or relapse (Fig. 1).

Considering only samples from the time of diagnosis, somatic variants in epigenetic regulators were found in $25 \%$ of $60 \mathrm{~B}-\mathrm{ALL}$ samples (Fig. 2), which was similar to de novo T-ALL patients ${ }^{5}$. In contrast, $50 \%$ of B-ALL samples harboured a mutation in a

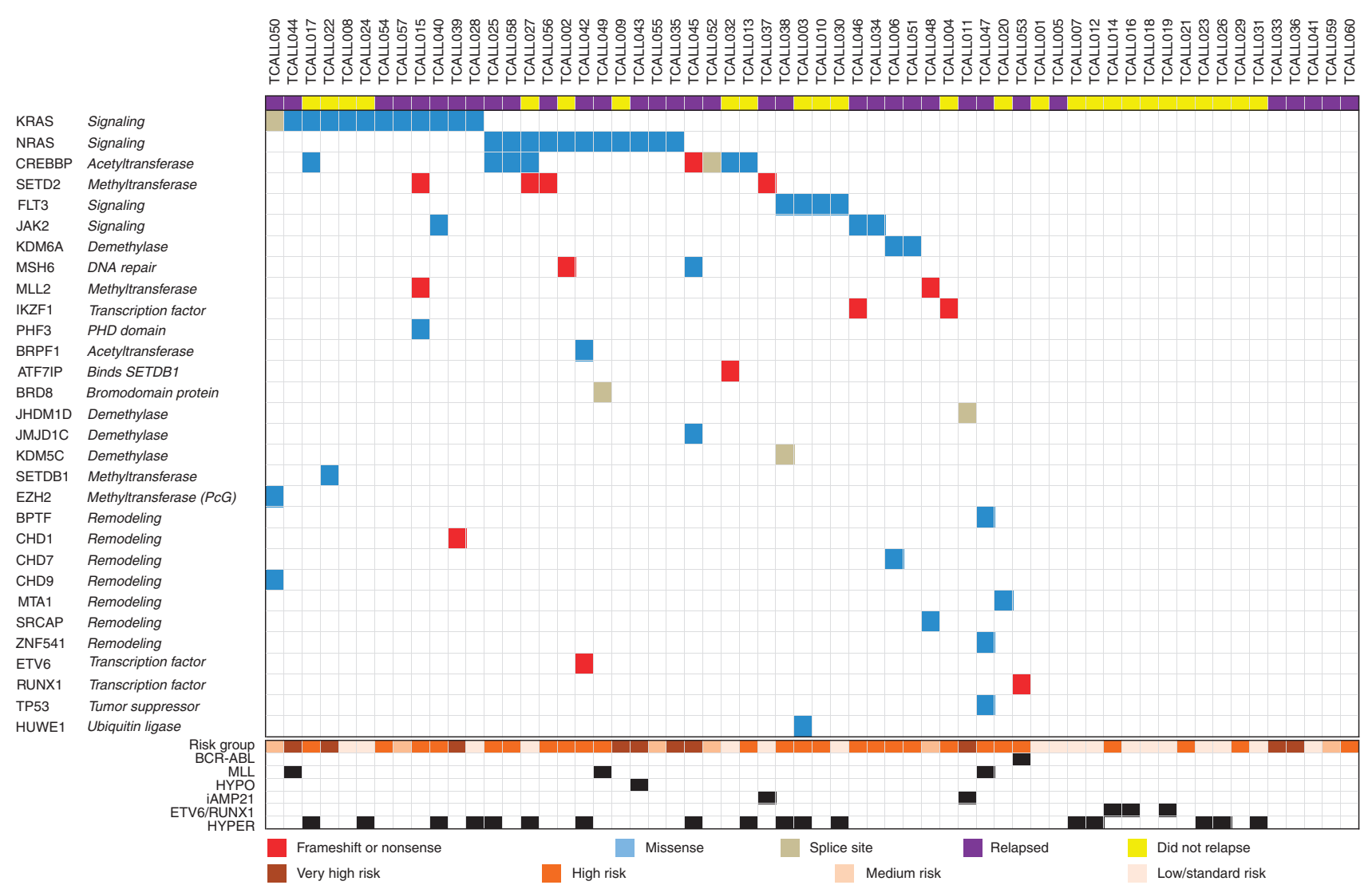

Figure 1 | Targeted hybrid capture sequencing identified somatic mutations in $\mathbf{7 0} \%$ of B-ALL patients. Matched tumour-remission samples from 60 paediatric B-ALLs were sequenced with targeted hybrid capture and somatic mutations in any tumour sample were identified. Genes on the left are organized by mutational frequency. The type of alteration is identified for each mutation as frameshift/stop, missense or splice site mutation. MLL, MLL rearranged; HYPO, hypodiploidy; iAMP21, intrachromosomal amplification of chromosome 21; HYPER, hyperdiploidy. 


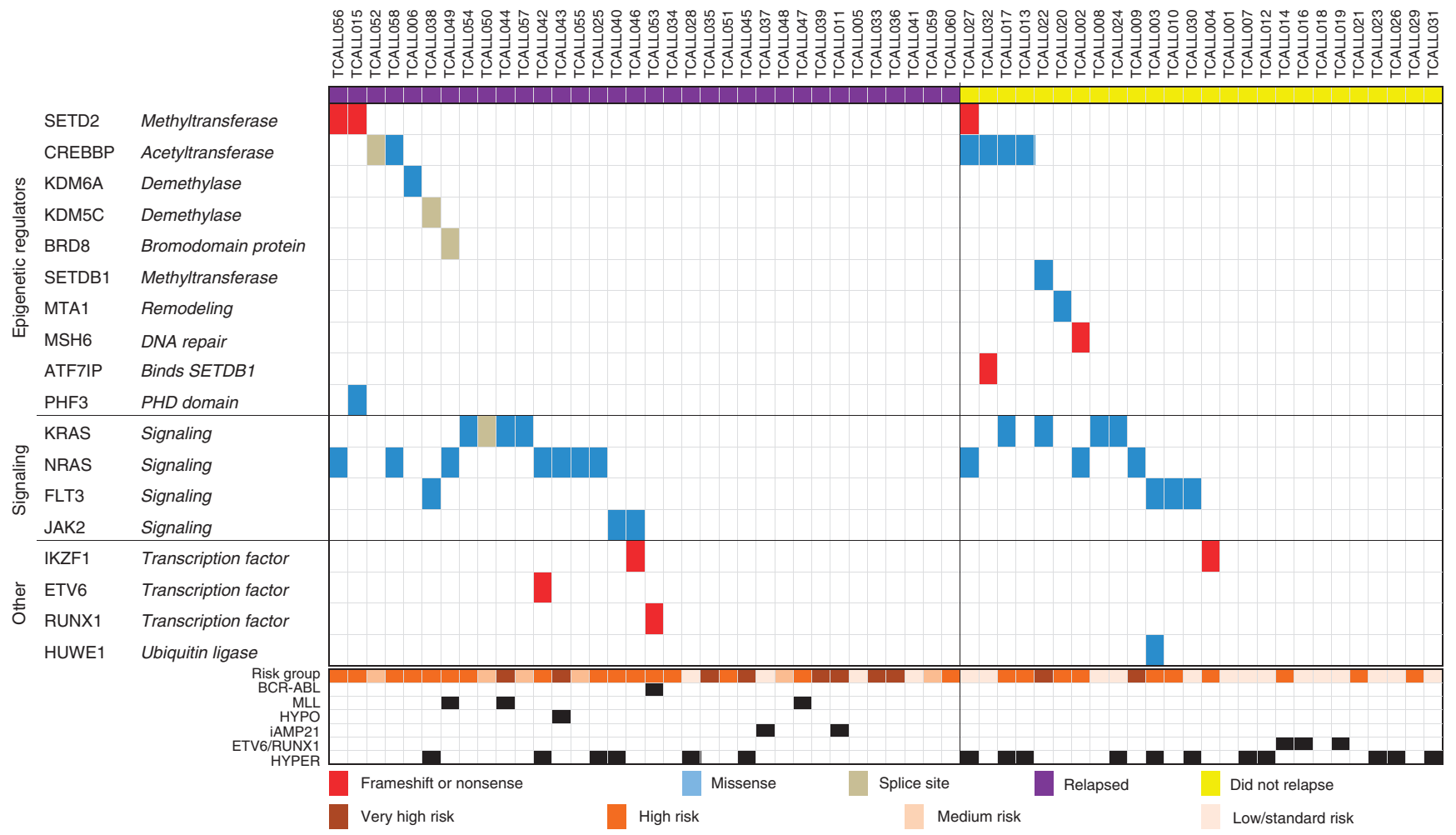

Figure 2 | Mutations in epigenetic regulators occur in 25\% of B-ALL patients at diagnosis, including SETD2. Matched diagnosis and remission sample pairs from 60 paediatric B-ALL patients were baited with target hybrid capture, Illumina sequenced and somatic mutations were identified. Patients are organized by those that eventually relapsed (on the left), and those that did not (on the right). Final risk category and molecular subtypes listed below. MLL, MLL rearranged, HYPO, hypodiploidy, iAMP21, intrachromosomal amplification of chromosome 21, HYPER, hyperdiploidy.

signalling factor at diagnosis with recurrent mutations in the Ras pathway, including activating point mutations in KRAS and NRAS, which were often subclonal (Supplementary Fig. 1A). At diagnosis, recurrent mutations in epigenetic regulator genes were identified in CREBBP (10\%) and SETD2 (5\%).

SETD2 mutations in de novo ALL. To more accurately determine the frequency of SETD2 mutations, which have not been previously described in B-ALL, the coding regions of SETD2 were PCR amplified and Illumina sequenced in a more representative, independent validation cohort of 125 de novo paediatric ALL patients. SETD2 mutations were found in $12 \%$ of the cohort and these leukaemias also frequently harboured mutations in the Ras pathway (Fig. 3a). Four patients harboured multiple SETD2 mutations. In total, we identified 24 SETD2 mutations in 19 out of 185 ALL patients, including seven loss-of-function frameshift or nonsense mutations, with no obvious hotspot mutations (Fig. 3b). Altogether, SETD2 mutations were most prevalent in the MLL rearranged (22\%) and ETV6-RUNX1 rearranged (13\%) subtypes of ALL (Supplementary Table 3). Of note, these two subtypes were underrepresented in the initial cohort, explaining the lower prevalence of SETD2 mutations in that cohort.

Enrichment of mutations in epigenetic regulators at relapse. Sequencing of the 30 matched relapsed samples showed a striking gain of mutations in epigenetic regulators, with 17 (57\%) harbouring a mutation. Of the 11 patients that gained a mutation in an epigenetic regulator at the time of relapse, only 3 were detectable in a rare subclone at diagnosis. (Fig. 4a). As noted above, this frequency is comparable to other poor prognosis de novo ALL subgroups, such as hypodiploid and ETP ALL. In addition to the 11 gains, 5 patients retained the same mutations from diagnosis, and 1 patient had their clonal mutation reduced to a subclonal allele frequency at relapse (Supplementary Fig. 1B). Details of each identified somatic mutation in each patient and a summary of mutations by molecular subtype are available in the Supplementary Materials (Supplementary Tables 3 and 4).

By McNemar test, there was a statistically significant enrichment of the entire epigenetic group $(P=0.0039)$ (Fig. $4 \mathrm{~b})$ and SETD2 and CREBBP together $(P=0.045)$. In contrast, there was not a significant enrichment of somatic mutations in signalling factors such as NRAS, KRAS, FLT3 and JAK2, as individual genes, or as a group. This was because there were as many patients gaining signalling mutations or mutational allele frequency as those losing them (six, McNemar test $P=1.00$ ) (Fig. $4 \mathrm{~b}$ ). The selective enrichment of epigenetic regulators is consistent with the hypothesis that mutations in genes encoding epigenetic regulators mediate chemotherapy resistance. Conversely, oncogenic mutations in signalling factors showed no enrichment, with frequent losses and gains, implying no direct role in modulating drug resistance and non-dependence on any particular mutation. Thus, solely targeting signalling factors by specific inhibition may not circumvent the development of chemoresistance. In total, recurrently mutated epigenetic regulators included $M L L 2$, KDM6A and MSH6, as well as additional mutations in SETD2 and CREBBP. KDM6A encodes for a H3K27 demethylase. We recently reported somatic KDM6A mutations in $4 \%$ of paediatric ALL at diagnosis, with mutations clustered in the catalytic jmjC domain $^{6}$. SETD2 is the sole mammalian H3K36 trimethyltransferase, and mutations in it have been described in ETP $\mathrm{T}_{-\mathrm{ALL}^{2}}$ and clear cell renal cell carcinoma ${ }^{7}$, but SETD2 has not been found to be recurrently mutated in large cohorts of non-ETP T-ALL ${ }^{2,5,8}$ and $\mathrm{AML}^{9}$ patients. 

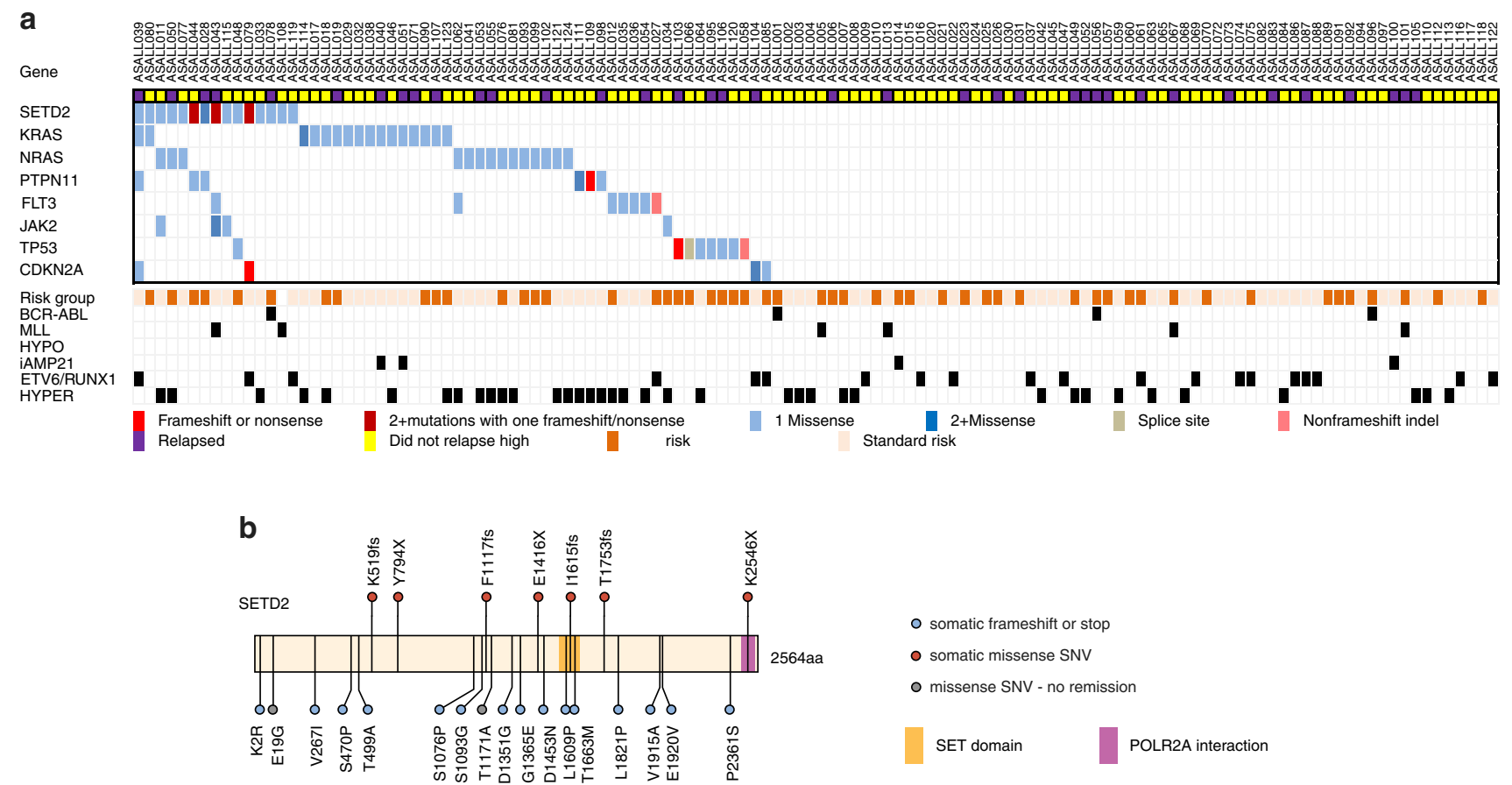

Figure 3 | SETD2 mutations in de novo ALL. (a) Amplification and Illumina sequencing of coding exons of SETD2 and other frequently mutated genes in an validation cohort of 125 de novo ALL patients identified 20 additional SETD2 mutations in 15 patients (12\%). Relapse status is noted above. Final risk category and molecular subtypes listed below. MLL, MLL rearranged; HYPO, hypodiploidy; iAMP21, intrachromosomal amplification of chromosome 21; HYPER, hyperdiploidy. (b) Schematic of somatic SETD2 mutations found in ALL patients.

MSH6 is a vital component of the DNA mismatch repair (MMR) machinery that localizes to chromatin by binding to the H3K36 trimethyl mark that SETD2 makes. SETD2 knockdown has been shown to cause mislocalization of MSH6 and microsatellite instability and a mutator phenotype in several cell types ${ }^{10}$.

\section{Discussion}

Here, we report that somatic mutations in epigenetic regulators are enriched at relapse in paediatric B-ALL with CREBBP, KDM6A, MLL2, SETD2 and MSH6 as recurrently mutated genes. This finding suggests that therapy may be applying pressure to acquire or select for rare subclones that possess these mutations. As noted above, SETD2 and MSH6 have been linked together in DNA MMR via the H3K36 trimethyl mark. We speculate that the loss-of-function mutations identified in SETD2 and MSH6 may lead to clonal survival by at least two possible mechanisms. One, a mutator phenotype induced by SETD2 inactivation could increase the mutational diversity and thus, adaptability of the leukaemia, leading to clonal survival. Two, since intact MMR is important for triggering apoptosis and/or cell cycle arrest in response to many DNA damaging chemotherapies, SETD2 loss may lead to chemotherapy tolerance. For example, leukaemia cells lines with defective MMR show chemoresistance to the thiopurines 6-MP and 6-TG ${ }^{11}$, which are commonly used in ALL therapy. These mechanisms are potentially reversible by inhibiting the epigenetic regulators that oppose SETD2 activity such as the H3K36 demethylases.

Interestingly, alterations in other factors which modify $\mathrm{H} 3 \mathrm{~K} 36$ methylation, such as NSD1, WHSC1/NSD2, NSD3 and KDM4A have been associated with ALL, AML, multiple myeloma, breast cancer and myelodysplastic syndrome ${ }^{12}$. Several recent reports have identified recurrent activating WHSC1/NSD2 E1099K mutations in many ALL cell lines and $20 \%$ of ETV6-RUNX1 translocated ALL patients ${ }^{13,14}$.
The genetic basis for chemoresistance and poor outcomes in relapsed ALL is beginning to be defined. For example, recent studies have identified relapse acquired NT5C2 mutations in ALL which lead to thiopurine resistance (ref 8,15). This study identifies mutations in genes encoding epigenetic regulators as frequently gained at the time of relapse. Although this study was not powered to determine a prognostic effect of these mutations in this modest cohort of patients, the enrichment of mutations in epigenetic regulators in relapsed B-ALL and other chemoresistant, high-risk subtypes of de novo leukaemia is quite suggestive of a role in clonal survival. Epigenetic regulators deserve particular attention because they are amenable to pharmacological modulation. Histone deacetylase inhibitors are currently in clinical use ${ }^{16}$, and histone methyltransferase inhibitors, such as DOT1L inhibitors ${ }^{17}$, are in clinical development. Although these epigenetic therapies are first being evaluated in the relapsed setting, our work also suggests that incorporating epigenetic therapies in upfront therapy, particularly in patients with identified mutations, could be a strategy to prevent the development of chemoresistance and relapse.

Further studies are warranted to define the prognostic value of mutations in epigenetic regulators in ALL, their effects on chemotherapy resistance and whether therapeutic modulation of H3K36 methylation levels is a viable therapeutic strategy to prevent and treat relapse in patients with leukaemia.

\section{Methods}

Patient materials. Subjects were paediatric acute lymphoblastic patients enrolled and banked on upfront therapy protocols DFCI 00-001, DFCI 05-001, AIEOP2000, AIEOP-2006 or Interfant-99 and their families each gave informed consent for future biological research on their banked samples under protocols approved by the Institutional Review Board at each participating institution and Dana Farber Cancer Institute. Bone marrow specimens were primarily used, but peripheral blood was used for remission samples, if no bone marrow sample was available. Diagnostic and relapse marrow samples had at least $60 \%$ blasts by morphology. Mononuclear cells from each sample were isolated with Ficoll density gradient centrifugation and genomic DNA was extracted. 
Evolution of mutations at relapse

SETD2 Methyltransferase

MLL2 Methyltransferase

EZH2 Methyltransferase (PcG)

CREBBP Acetyltransferase

BRPF1 Acetyltransferase

KDM6A Demethylase

JHDM1D Demethylase

을 JMJD1C Demethylase

$\frac{\pi}{5}$ KDM5C Demethylase

BPTF Remodeling

C Remodeling

응 $\mathrm{CHD7} \quad$ Remodeling

CHD9 Remodeling

SRCAP Remodeling

ZNF541 Remodeling

MSH6 DNA repair

BRD8 Bromodomain protein

PHF3 PHD domain

KRAS Signaling

NRAS Signaling

듄 SLT3 Signaling

के JAK2 Signaling

IKZF1 Transcription factor

¿ ETV6 Transcription factor

ठ̃ RUNX1 Transcription factor

TP53 Tumor suppressor

b
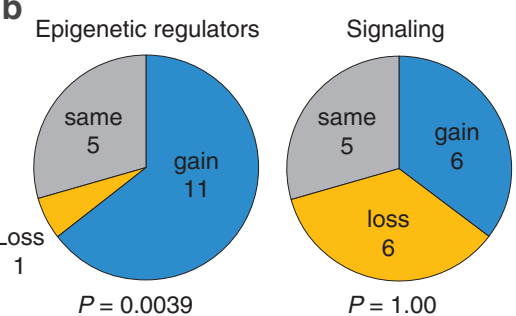

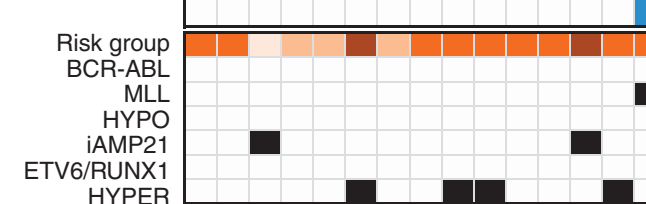

Gained at relapse, undetected at diagnosis

Gained from subclone at diagnosis

Similar clone at relapse

Very high risk

High risk

Medium risk

Lost completely at relapse

Significant loss, but detectable

$P=1.00$

Figure 4 | Relapsed B-ALL is enriched for mutations in epigenetic regulators. (a) Gain or loss of somatic mutations in matched diagnosis-relapse samples from 30 paediatric B-ALL patients. Clonal mutations at relapse which were detected in a minor subclone at diagnosis and clonal mutations at diagnosis which became subclonal at relapse are shaded. MLL, MLL rearranged; HYPO, hypodiploidy; iAMP21, intrachromosomal amplification of chromosome 21; HYPER, hyperdiploidy. (b) Comparison of the number of patients which gain, lose or have the same number of mutations in epigenetic regulators and signalling factors at relapse. The McNemar test was used to calculate the $P$-values.

Exon capture. For exon capture, $1-2 \mu \mathrm{g}$ of genomic DNA was sheared with a Covaris E100 to an average size of $200 \mathrm{bp}$. Sheared DNA was end repaired, a-tailed and ligated to barcoded adaptors as per the Illumina Truseq Library Preparation protocol. Libraries were amplified with the Kapa Realtime Library Amplification kit to minimize the number of PCR cycles used (range 4-12). Library was then quantified by Kapa Realtime Quantification Kit and pooled up to six samples per lane in equimolar amounts totalling up to $500 \mathrm{ng}$ of DNA. Each pool was then hybridized to a custom SureSelect In Solution Hybrid Capture RNA baitset, consisting of 19,952 baits, spanning $2.35 \mathrm{Mb}$ and 472 genes (Supplementary Table 1). Each capture was washed and amplified with the Kapa Realtime Library Amplification Kit and submitted for sequencing on an Illumina HiSeq 2000100 bp singleend run.

Amplicon sequencing. 192 primer pairs spanning the coding regions of SETD2, NRAS, KRAS, FLT3, JAK2, PTPN11, TP53 and CDKN2A were designed and pooled into 48 primer pools and used for amplification in the Fluidigm All Access Array (Supplementary Table 5). $50 \mathrm{ng}$ of genomic DNA samples from 125 patients, 48 samples at a time with 48 primers pools, were amplified by the Fluidigm
All Access microfluidics system and each tagged with a unique barcode. All samples were then pooled and sequenced on one Illumina Hiseq $2000100 \mathrm{bp}$ single-end run.

Variant calling. Fastg files were aligned to hg19 version of the human genome with BWA. SNP, indel and CNV calling was performed with samtools mpileup and Varscan 2.2.3 somatic option. Annovar was used to annotate variants. Relapsed and diagnostic variants were linked and they were filtered for $\geq 15 \%$ allele frequency and $20 \times$ coverage in either tumour sample, $<5 \%$ and $10 \times$ coverage in the remission sample, bidirectional variant reads, minimal strand bias and minimal local noise. Variants were visualized in the Integrated Genome Viewer to eliminate variants with clustered read position and variants caused by indel artifacts. Sanger sequencing was used to validate a sample of somatic variants found by next generation sequencing to validate filtering criteria. Every filtered somatic variant position was then forced called on every sample to examine the baseline noisiness of each position. Those with mean baselines $>1 \%$ allele frequency in remission samples were excluded. Variants were also filtered to only those positions within $50 \mathrm{bp}$ of those included in the custom capture baitset. Variants were considered 
somatic if they were present in $<5 \%$ allele frequency in the paired remission sample (to account for high minimal residual disease).

\section{References}

1. Mullighan, C. G. et al. Genomic analysis of the clonal origins of relapsed acute lymphoblastic leukemia. Science 322, 1377-1380 (2008).

2. Zhang, J. et al. The genetic basis of early T-cell precursor acute lymphoblastic leukaemia. Nature 481, 157-163 (2012).

3. Holmfeldt, L. et al. The genomic landscape of hypodiploid acute lymphoblastic leukemia. Nat. Genet. 45, 242-252 (2013).

4. Mullighan, C. G. et al. CREBBP mutations in relapsed acute lymphoblastic leukaemia. Nature 471, 235-239 (2011).

5. De Keersmaecker, K. et al. Exome sequencing identifies mutation in CNOT3 and ribosomal genes RPL5 and RPL10 in T-cell acute lymphoblastic leukemia. Nat. Genet. 45, 186-190 (2013).

6. Mar, B. et al. Sequencing histone-modifying enzymes identifies UTX mutations in acute lymphoblastic leukemia. Leukemia. 26, 1881-1883 (2012).

7. Dalgliesh, G. et al. Systematic sequencing of renal carcinoma reveals inactivation of histone modifying genes. Nature 463, 360-363 (2010).

8. Tzoneva, G. et al. Activating mutations in the $\mathrm{NT5C} 2$ nucleotidase gene drive chemotherapy resistance in relapsed ALL. Nat. Med. 19, 368-371 (2013).

9. Cancer Genome Atlas Research Network. Genomic and epigenomic landscapes of adult de novo acute myeloid leukemia. New Engl. J. Med. 368, 2059-2074 (2013)

10. Li, F. et al. The histone mark H3K36me3 regulates human dna mismatch repair through its interaction with MutSalpha. Cell 153, 590-600 (2013).

11. Diouf, B. et al. Somatic deletions of genes regulating MSH2 protein stability cause DNA mismatch repair deficiency and drug resistance in human leukemia cells. Nat. Med. 17, 1298-1303 (2011)

12. Wagner, E. \& Carpenter, P. Understanding the language of Lys36 methylation at histone H3. Nat Rev Mol Cell Biol 13, 115-126 (2012).

13. Jaffe, J. D. et al. Global chromatin profiling reveals NSD2 mutations in pediatric acute lymphoblastic leukemia. Nat. Genet. 45, 1386-1391 (2013).

14. Oyer, J. et al. Point mutation E1099K in MMSET/NSD2 enhances its methyltranferase activity and leads to altered global chromatin methylation in lymphoid malignancies. Leukemia. 28, 198-201 (2013).

15. Meyer, J.A. et al. Relapse-specific mutations in NT5C2 in childhood acute lymphoblastic leukemia. Nat Genet 45, 290-294 (2013).
16. Spiegel, S., Milstien, S. \& Grant, S. Endogenous modulators and pharmacological inhibitors of histone deacetylases in cancer therapy. Oncogene 31, 537-551 (2012)

17. Daigle, S. et al. Selective killing of mixed lineage leukemia cells by a potent small-molecule DOT1L inhibitor. Cancer Cell 20, 53-65 (2011).

\section{Acknowledgements}

We are grateful to C. Lindsley, S. Jaiswal, A. Krivtsov, M. Correll, R. Rubio,

F. Abderazzaq and Y. Wang for technical advice and valuable discussions. We would also like to thank S. Hunt, E. Swaim, J. Allen, H. Wang and A. Lack for assistance in obtaining clinical specimens. B.G.M. was supported by the National Institutes of Health (5T32CA136432-03), the Lady Tata Memorial Trust, the William Lawrence and Blanche Hughes Foundation, the Abraham Fellowship Fund and the Friends of Dana Farber. Additional research support was provided by the National Institutes of Health (CA140575 and CA068484), the Charles H. Hood Foundation and the Leukemia and Lymphoma Society.

\section{Author contributions}

B.G.M., L.B.B. and S.A.A. conceived and designed the project. K.M.M. and B.G.M. performed the sequencing library preparation and preparing of samples for Sanger sequencing. B.G.M. and P.V.G. performed the informatics analysis of the sequencing data. M.H.H., L.B.S., S.E.S. and L.L.N. provided patient samples for analysis. K.S. and D.S.N. provided clinical annotation and biostatistical analysis. B.G.M. wrote the manuscript and S.A.A., L.B.B. and B.L.E. edited the manuscript. S.A.A. and B.L.E. jointly directed the work.

\section{Additional information}

Supplementary Information accompanies this paper at http://www.nature.com/ naturecommunications

Competing financial interests: The authors declare no competing financial interests.

Reprints and permission information is available online at http://npg.nature.com/ reprintsandpermissions/

How to cite this article: Mar, B. G. et al. Mutations in epigenetic regulators including SETD2 are gained during relapse in paediatric ALL. Nat. Commun. 5:3469 doi: $10.1038 /$ ncomms4469 (2014). 